\title{
Spread Spectrum for Chaotic Compressed Sensing Techniques in Parallel Magnetic Resonance Imaging
}

\author{
Tran Duc - Tan, Le Vu-Ha, and Nguyen Linh - Trung \\ University of Engineering and Technology, Vietnam National University, Hanoi, Vietnam
}

\begin{abstract}
We consider the fast acquisition problem in magnetic resonance imaging (MRI). Often, fast acquisition is achieved using parallel imaging (pMRI) techniques. It has been shown recently that compressed sensing (CS), which enables exact reconstruction of sparse or compressible signals from a small number of random measurements, can accelerate the speed of MRI acquisition because the number of measurements was small. Recently, the spread spectrum (SS) has been utilized to enhance the quality of reconstructed CS image. Also recently, chaotic CS approach potentially offers simpler hardware implementation. In this paper, we combine chaotic CS and spread spectrum technique in order to obtain a fast acquisition in MRI with enhanced quality of reconstructed image. The performance of the proposed method is analyzed using numerical simulation.
\end{abstract}

Key words - fast acquisition, magnetic resonance imaging (MRI), compressed sensing (CS), deterministic chaos,

\section{INTRODUCTION}

Magnetic Resonance Imaging (MRI) has found various applications in the field of biology, engineering, and material science. Fast image acquisition in MRI is important in order to enhance image contrast and resolution, to avoid physiological effects or scanning time on patients, to overcome physical constraints inherent within the MRI scanner, or to meet timing requirements when imaging dynamic structures or processes.

State-of-the-art techniques for fast MRI are mainly in the form of parallel imaging in which multiple coils are simultaneously used. Each coil acquires data corresponding to a portion of the imaging object. There exists some redundancy in the acquired data across all the coils. While the acquisition time is inversely proportional to the number of coils, it is this redundancy that can be exploited to reconstruct the final object image. The reconstruction of the image can be done in the image domain, the k-space domain or the k-t-space domain. In the image domain approach, image reconstruction is done by solving a set of linear equations in the image domain. A common technique is SENSE (SENSitivity Encoding) [1] which uses the sensitivity profiles in order to reduce the acquisition time. SENSE-like methods include SPACE-RIP [2] and PILS (Parallel Imaging with Localized Sensitivities) [3]. The k-space domain approach uses partial data obtained in all the coils to synthesize the full $\mathrm{k}$-space, hence reconstruct the MRI image [4][5]. In the k-t-space domain approach, the k-t SENSE method [6] exploits correlations in both k-space and time. The UNFOLD (UNaliasing by Fourier-encoding the Overlaps Using the temporal Dimension) method [7] encodes the sensitivity into pre-determined frequency bands. $k-t$ SENSE method can be applied to arbitrary k-space trajectories, time-varying coil sensitivities, and various reconstruction problems.

A recent breakthrough in mathematics and signal processing under the name of compressed sensing (CS) shows that sparse or, more generally, compressible signals can be recovered from a small number of linear random measurements [9]. Exact reconstruction can be achieved by nonlinear algorithms, using such as $l_{l}$ norm or Orthogonal Matching Pursuit [10]. In the context of signal sampling, CS is seen as random undersampling. This method is important because many signals of interest, including natural images, diagnostic images, videos, speech and music, are sparse in some appropriate domain of signal representation. Among various applications of $\mathrm{CS}$, it has recently been shown to be successfully applied to MRI for fast acquisition by Lustig et al. in [11]. In particular, random undersampling is carried out in the k-space. In other words, by acquiring the image with a smaller number of measurements as compared to normal full sampling, the speed of acquisition can be enhanced. Note that if we combine CS and parallel imaging, the speed will be further enhanced. Inspired by this work, further developments in the direction of using CS for MRI continues [12],[17],[18].

In CS, random measurement process is often used, providing a mathematical convenience for proving exact reconstruction of the signals. This naturally poses a question: can the measurement process be designed deterministically? As an answer to this question, recently, chaotic CS has been proposed in the form of a chaos filter by Linh-Trung et al. [13]. Chaos is aperiodic long-term behavior in a deterministic system that exhibits sensitive dependence on initial conditions. The system is so nonlinear that the output quickly becomes random-like. The proposed chaos filter was numerically showed to perform signal reconstruction better than random filters while enjoying a potential benefit of simple hardware design for chaotic generator as opposed to random generator. In practice, a "random" sequence is generated by a periodic pseudo-random generator, realized by a feedback shift register. A long register is needed to make the period long for the sequence to be more "random", and hence a large storage capacity and logic circuits are needed [14].

Recently, Puy et al. [16] have proposed a fast MRI acquisition system that pre-modulated signals by using quadratic phase profiles. Thus, the spread spectrum (SS) effect has been created in order to enhance the quality of the reconstructed image. However, this work has only focused to uniform random sampling in the $\mathrm{k}$-space that leads to impractical system. 
In this paper, we apply the method in [1], [13], and [16] to parallel MRI (pMRI) for fast acquisition and compare it with the chaotic CS method developed in [17]. The paper is organized as follows. In Section 2, we introduce the principle of compressed sensing theory, pMRI acquisition, pMRI using compressed sensing, and spread spectrum technique in the role of enhancing the quality of images. Section 3 presents our proposed method for pMRI acquisition by chaotic measurements and spread spectrum phenomenon. Simulation results that demonstrate the efficiency of our method are presented in Section 4. Section 5 concludes the paper with discussions on the results.

\section{BRIEF BACKGROUND}

\section{A. Chaotic compressed sensing}

Let $x \in R^{N}$ be the signal of interest and suppose that we know $x$ admits a sparse linear representation which reads $\mathrm{x}=\Phi \mathrm{s}$, where $s \in R^{N}$ is a $K$-sparse vector (i.e., containing exactly $K$ nonzero values) and $\Phi \in R^{N \times N}$ is called the sparsifying matrix. Suppose also that we measure/sense $x$ by a linear system $\Psi \in R^{M \times N}$, called the measurement matrix. Then, the measurements are given by $\mathrm{y}=\Psi \mathrm{x}$, with $y \in R^{M}$. Suppose we want to reconstruct $x$ from $y$. This is equivalent to reconstructing $s$ from $y$, since we can write $\mathrm{y}=\Theta \mathrm{s}$, where $\Theta=\Psi \Phi$.

A problem of tremendous interest, called compressed sensing (CS), is when $M$ is considerably less than $N$. The system $\Psi$ or, equivalently $\Theta$, becomes underdetermined. Thus, CS has two main tasks: (i) measurement (encoding) - how to design the measurement system $\Psi$ to obtain the measurement $y$, and (ii) reconstruction (decoding) - how to faithfully reconstruct $x$ from $y$. We wish to have $M$ as small as possible and the reconstruction algorithm as efficient as possible.

If the sparsity information in $x$ is still fully kept, though hidden, in $y$, exact reconstruction of $s$ is feasible if we find a way to fully restore this sparsity from y. Thanks to the sparse structure of $\mathrm{s}$, the exact reconstruction of the signal is made possible when $\Theta$ is constructed as an almost orthonormal system when restricted to sparse linear combinations and satisfies sufficient conditions called Restricted Isometry Properties (RIPs).

A useful indicator for this property is the measure of incoherence. $\Phi$ is incoherent with $\Psi$ in the sense that one can not sparsify the other [15]. One way to ensure the incoherence is to have $\Psi$ as a random matrix with Gaussian i.i.d. elements. Under such a condition, $s$ can be faithfully recovered from $y$ when $M$ is such that $\mathrm{cK} \cdot \log (\mathrm{N}=\mathrm{K})<\mathrm{M}<\mathrm{N}$, where $c$ is some constant, using various sparse approximation techniques, for examples, $l_{1}$-optimization based Basis Pursuit (BP) [1] or Orthogonal Matching Pursuit (OMP) [10].

In a recent paper [13], we proposed to use a chaotic measurement matrix $\Psi$, which is deterministic, instead of random one. To construct the chaotic measurement matrix $\Psi$, generate sampled logistic sequence by a deterministic chaotic system, then create the matrix $\Psi$ column by column with this sequence. Elements of the logistic sequence are generated by deterministic chaotic system which is so nonlinear, hence becomes random-like. After that, the reconstruction is also performed by the OMP technique. There, the simulated results indicated that the chaotic approach outperformed the random approach in terms of the probability of exact reconstruction. Moreover, using chaotic CS system also inherits a simpler hardware implementation compared to the random one.

\section{B. Parallel Imaging based on SENSE}

The number of excitations, i.e. the number of horizontal lines in the k-space trajectory as shown in Fig. 1, determines the total acquisition time. In SENSE, the number of horizontal lines in the trajectory traced by each individual coil is reduced by the number of coils in use. Subsequently, the sensed size of the imaged area is also reduced. The spatial resolution is not changed but aliasing artifacts appear.

SENSE works in the image domain by removing the aliasing effect caused by combining the individual images, called field-of-view (FOV) images, obtained by individual coils. The inversion of the aliasing transformation for each pixel is calculated individually. Consider the imaging of a slice of the object in the 2D plane $(x ; y)$. Let $m(x ; y)$ be this image. Let $L$ be the number of RF coils. Each coil would have individual values of image intensity. The $\mathrm{k}$-space signal obtained from the $l$-th coil is given by:

$$
s_{l}\left(k_{x}, k_{y}\right)=\iint_{x y} C_{l}(x, y) m(x, y) e^{-i 2 \pi\left(k_{x} x+k_{y} y\right)} d x d y
$$

where $\mathrm{C}_{1}(\mathrm{x}, \mathrm{y})$ is the sensitivity function of the $l$-th coil; $k_{x}$ and $k_{y}$ encode the information of location along the $x$ and $y$ directions of the image respectively, and $k=\left\{k_{x}, k_{y}\right\}$ is said to lie in the k-space.

Equation (1) shows that $\mathrm{s}_{\mathrm{l}}\left(\mathrm{k}_{\mathrm{x}}, \mathrm{k}_{\mathrm{y}}\right)$ is the Fourier transform of the sensitivity-weighted images $\mathrm{C}_{\mathrm{l}}(\mathrm{x}, \mathrm{y}) \mathrm{m}(\mathrm{x}, \mathrm{y})$. The image acquired by each individual coil $\mathrm{m}_{\mathrm{l}}(\mathrm{x}$; $\mathrm{y})$ can then be expressed as the ideal image modulated by the corresponding sensitivity function:

$$
m_{l}(x, y)=C_{l}(x, y) m(x, y)
$$

Subsequently, each pixel of the full FOV image can be estimated as:

$$
\widehat{m}(x, y)=C^{H}(x, y) C^{-1}(x, y) C^{H}(x, y) m(x, y)
$$

where $\mathbf{C}=\left[\mathrm{C}_{1}, \ldots, \mathrm{C}_{\mathrm{L}}\right]$. In practice, a calibration procedure with the reference images is used to measure the sensitivity of each coil. These reference images must not contain aliasing artifact and noise. Smoothing and extrapolation of the coil sensitivity can be done to obtain an acceptable sensitivity map.

Fig. 1 presents the k-space of an MR image of a brain slice, which is shown in Fig. 4. We see that most of the encoded 
information concentrates around the origin of the k-space. In practice, the density of the k-space follows a power law. We can see from Fig. 1 that most encoded information is concentrated at the origin. In practice, the density of the kspace follows a power law (see Fig. 2).
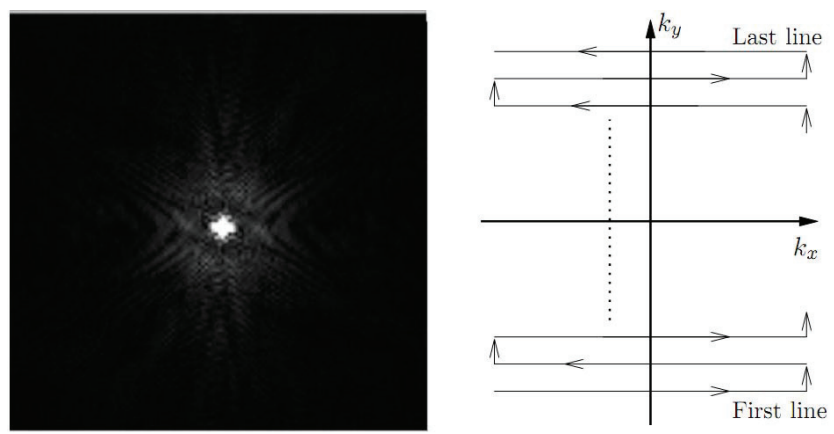

Fig. 1. k-space of a brain MR image and a full linear sampling trajectory.

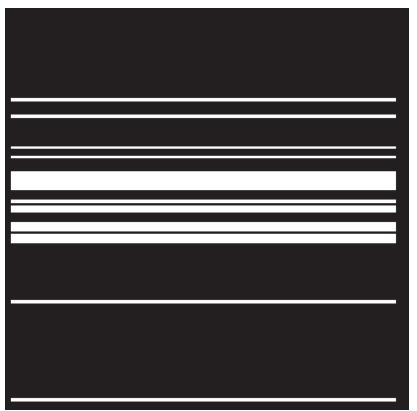

Fig. 2. Binary mask $(128 \times 128$ point $)$ illustrate the chaotic under-sampling performed in the k-space following the power law with the ratio of 0.15 . Note that the spatial frequencies are assumed to be acquired as the light discrete points.

\section{Multichannel compressed sensing using conjugate gradient SENSE}

The chaotic measurement matrix is formed in MRI acquisition procedure. We generate the values of $k_{x}$ and $k_{y}$ by a logistic map process, and a couple of $\mathrm{k}_{\mathrm{x}}$ and $\mathrm{k}_{\mathrm{y}}$ will determine a coordinate in the k-space that will be acquired. However, the distribution of information in $\mathrm{k}$-space concentrates nearby the origin and decays when $\mathrm{k}_{\mathrm{x}}$ and $\mathrm{k}_{\mathrm{y}}$ increase. Fig. 1 shows that most encoded information is concentrated at the origin. Therefore, we convert the distribution of logistic map sequence to Gaussian distribution. The reconstruction is obtained by solving the constrained optimization problem [11]:

$$
\begin{aligned}
& \arg \min _{m}\left\{\left\|F_{u} m-y\right\|_{2}^{2}+\lambda\|\Phi m\|_{1}\right\} \\
& \text { subject to }\left\|F_{u} m-y\right\|_{2}<\varepsilon
\end{aligned}
$$

where $y$ are $\mathrm{k}$-space measurements, $\mathrm{F}_{\mathrm{u}}$ is the undersampled Fourier operator associated with measurements, $\Phi$ is the sparsifying transform operator, and $\lambda$ is a data consistency tuning constant.

\section{Accelerated pMRI using spread spectrum technique and} CS

The key point of this method is pre-modulating the image $m$ by a linear chirp signal $\mathrm{P}(\mathrm{x}, \mathrm{y})=\exp \left[\mathrm{i} \pi\left(\mathrm{w}_{\mathrm{x}} \mathrm{x}^{2}+\mathrm{w}_{\mathrm{y}} \mathrm{y}^{2}\right)\right]$ before under-sampling in the k-space [16]. The chirp modulation can be experimentally implemented with a MR scanner. By varying the intensity of the quadratic field and the echo time, we can control the average of chirp rate $\left(\mathrm{w}_{\mathrm{x}}, \mathrm{w}_{\mathrm{y}}\right)$. Figure 3 illustrates of a specific chirp signal which would be utilized in simulation.
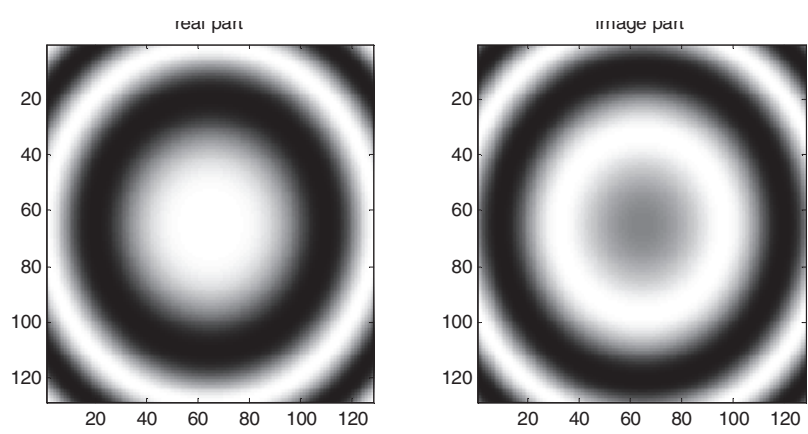

Fig. 3. Real and imaginary parts of a specific chirp signal at rate of $0.49 \mathrm{e}-3$.

The modulation spreads the spectrum of $m$ so that information of these vectors can be easily accessed in the kspace. In this method, magnetic resonance measurements in (1) can be re-written as:

$$
v_{l}\left(k_{x}, k_{y}\right)=\iint_{x y} m_{l}(x, y) e^{i \pi\left(w_{x} x^{2}+w_{y} y^{2}\right)} e^{-i\left(k_{x} x+k_{y} y\right)} d x d y(5)
$$

The advantage of using spread spectrum in this case is that the coherence between $\Phi$ and the Fourier basis F can be reduced hence enhance reconstruction quality [16]. In the previous literature of compressed sensing we have got $\Theta=\Psi \Phi$. In this propose, we have $\Theta=\Psi \boldsymbol{P} \Phi$ where $\boldsymbol{P}$ is chirp modulation matrix. The data is also reconstructed by solving the BP [11] or TV minimization [15].

\section{Chaotic Compressed SENSING USING SPREAD SPECTRUM TECHNIQUE}

The chirp modulation is applied in every channel by using quadratic profile in order to excite spread spectrum effect. Consequently, the chaotic measurement matrix is then applied to MRI acquisition procedure. We generate the values of $k_{x}$ and $k_{y}$ by a logistic map process, and a pair of $k_{x}$ and $k_{y}$ values will determine a coordinate in the $\mathrm{k}$-space that will be acquired. The reconstruction is obtained by solving the constrained optimization problem:

$$
\begin{aligned}
& \widehat{m}_{P}=\arg \min _{m}\left\{\left\|F_{u} P m-y\right\|_{2}^{2}+\lambda\|\Phi P m\|_{1}\right\} \\
& \text { subject to } \quad\left\|F_{u} P m-y\right\|_{2}<\varepsilon
\end{aligned}
$$

where $\hat{m}_{P}$ is modulation signal and $\boldsymbol{P}$ is chirp modulation 
matrix.

Once the MRI data has been acquired, the reconstruction is performed by the Nonlinear Conjugate Gradient (NCG) algorithm. Our scheme can be summarized in Algorithm 1.

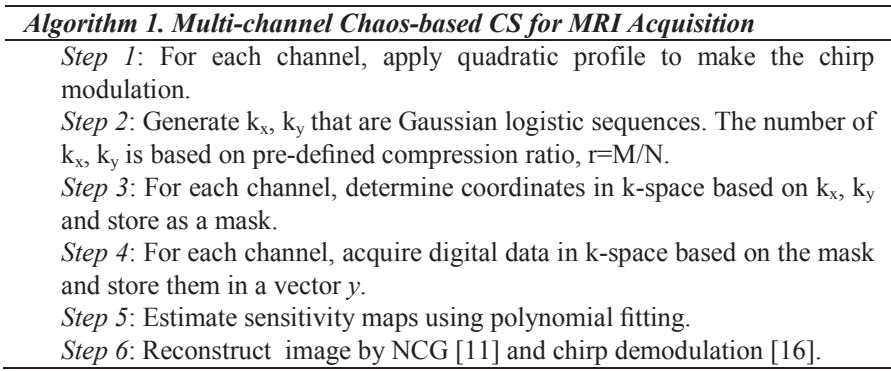

\section{RESUlt AND PERFormANCE}

In the simulation, the data source in use, obtained from [20], is human MPRAGE data from 8-channel head array coil. The data was acquired with the following parameters: $T E=$ $3.45 \mathrm{~ms}, T R=2350 \mathrm{~ms}, T I=1100 \mathrm{~ms}$, Flip angle $=7 \mathrm{deg}$, slice $=1$, matrix $=128 \times 128$, slice thickness $=1.33 \mathrm{~mm}, F O V=$ $256 \mathrm{~mm}$.

Under-sampling with the ratio of 0.30 is performed in the $\mathrm{k}$ space by the logistic map [13]. The chirp rate is assumed to be controlled at the value of $0.49 \mathrm{e}-3$. It can be seen that the chaotic CS with spread spectrum offer a higher quality of reconstruction image than chaotic CS without spread spectrum (see Fig. 4).

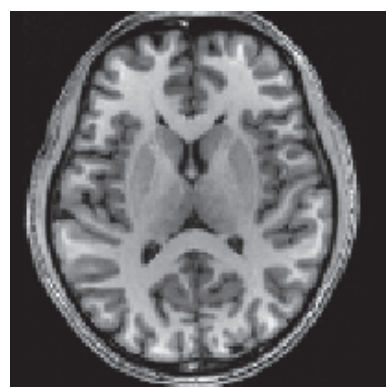

(a)

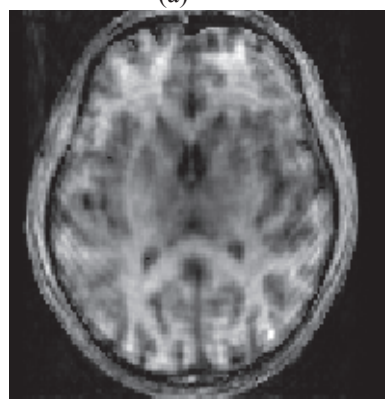

(c)

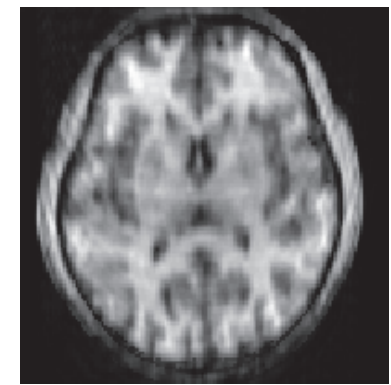

(b)

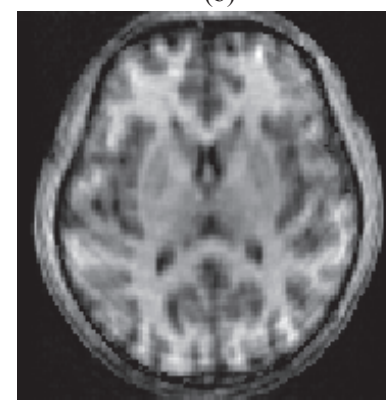

(d)
Fig. 4. MPRAGE phantom: (a) Original brain slice, (b) The CS image reconstructed by zero filling, (c) Chaotic CS with $r=0.15$ and without SS (d) Chaotic CS with $r=0.15$ with SS.

Fig. 5 shows Fourier transforms of the original (a) and modulated images (b). It can be seen that by using the chirp modulation, the signal spectrum is spread in the k-space that lead to an easier under-sampling.

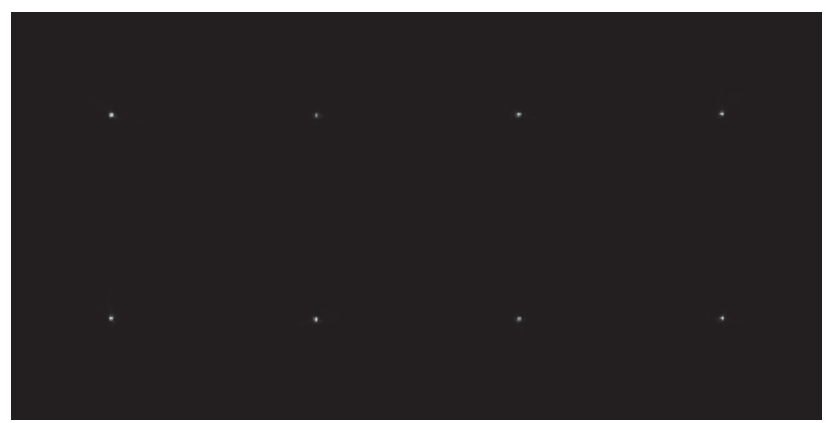

(a)

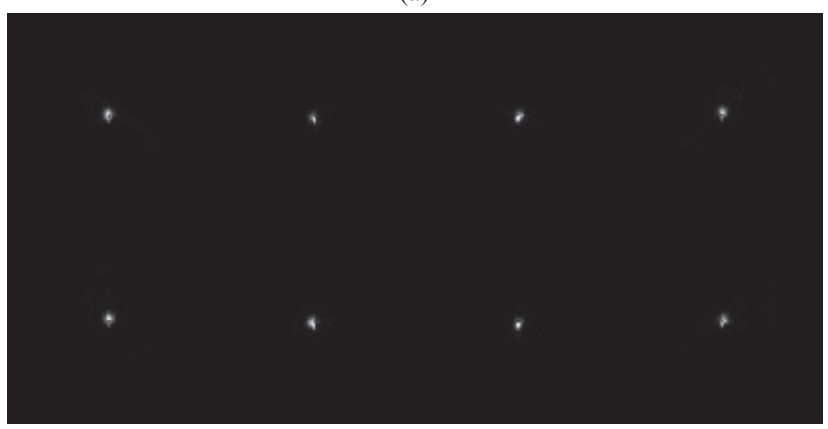

(b)

Fig. 5. Fourier transforms of the original (a) and modulated signals (b) in 8 channels.

To compare the efficiency of the proposed method, we acquire brain MRI data for a series of compression ratios by measurements with and without spread spectrum (see Fig. 6). Then, we determine, for each compression ratio, the error in the reconstructed image as compared to the original image. Suppose that $m$ is an $\mathrm{N} \times \mathrm{M}$ original image and $\hat{m}$ is the reconstructed image. The error can be defined as:

$$
\varepsilon=\frac{1}{N \times M} \sum_{i=1}^{N} \sum_{j=1}^{M}\left|m_{i j}-\hat{m}_{i j}\right|
$$

It is seen that spread spectrum offer better performance, especially in the low range of compression ratio. With a small value of compression ratio, the modulation spreads the spectrum of $m$ so that information of these vectors can be easily accessed in the $\mathrm{k}$-space. With a higher ratio, the role of parallel coils is obvious when the information in k-space from multi channels can be combined in reconstruction process. 


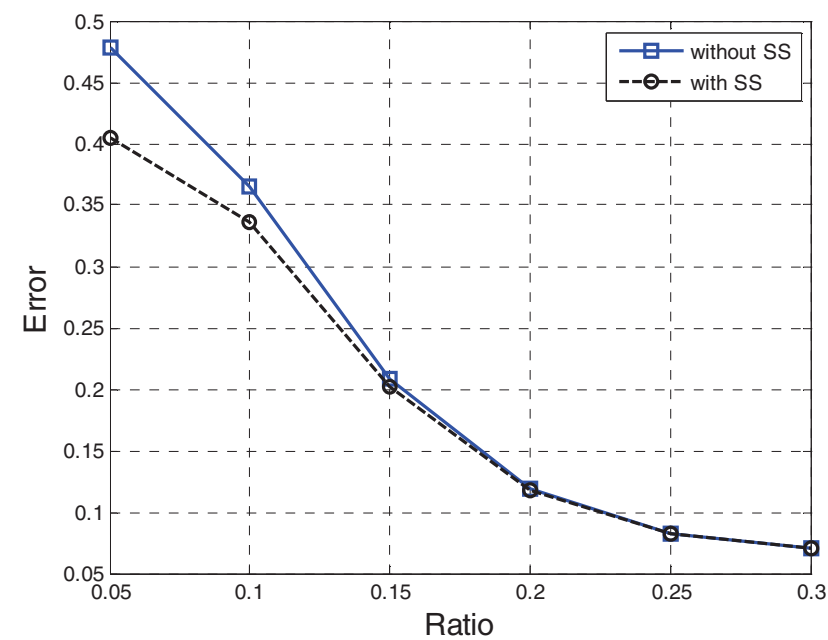

Fig. 6. Performance comparison between chaotic CS with and without SS.

\section{CONCLUSION}

Based on the works of [18] using chaotic CS, [17] and [19] using parallel MRI, and [16] using spread spectrum for 2D brain MRI images, we presented an application to a fast and practical acquisition scheme. Our results confirm the success of using chaotic measurements and the spread spectrum techniques for pMRI acquisition. Lastly, this approach outperforms not only the random CS proposed by Linh-Trung [18], Lustig \& Pauly [11], and Tan [17] in terms of error performance in image reconstruction but also the spread spectrum proposed by Puy [16] in terms of hardware implementation.

\section{ACKNOWLEDGMENT}

This research was supported by Asia Research Center, under project "Advanced signal processing techniques for improving the acquisition speed in magnetic resonant imaging". This work is partly supported by the Vietnam National Foundation for Science and Technology Development (NAFOSTED).

\section{REFERENCES}

[1] K. Pruessmann, M. Weiger, M. Scheidegger, and Boesiger, "SENSE: Sensitivity encoding for fast MRI," Magnetic Resonance in Medicine, vol. 42 , pp. $952-962,1999$

[2] W. E. Kyriakos, L. P. Panych, D. F. Kacher, C. F. Westin, S. M. Bao, R. V. Mulkern, and F. A. Jolesz, "Sensitivity profiles from an array of coils for encoding and reconstruction in parallel (SPACE RIP)," Magnetic Resonance in Medicine, vol. 44, no. 2, pp. 301-308, Aug 2000.

[3] M. Griswold, P. Jakob, M. Nittka, J. W. Goldfarb, and A. Haase, "Partially parallel imaging with localized sensitivities (PILS)," Magnetic Resonance in Medicine, vol. 44, pp. $602-609,2000$.

[4] D. Won and W. J. Manning, "Simutaneous acquisition of spatial harmonics (SMASH): fast imaging with radio frequency coil arrays,' Magnetic Resonance in Medicine, vol. 38, pp. 591 - 603, 1997.

[5] M. A. Griswold, P. M. Jakob, R. M. Heidemann, M. Nittka, V. Jellus, J. Wang, B. Kiefer, and A. Haase, "Generalized autocalibrating partially parallel acquisitions (GRAPPA)," Magnetic Resonance in Medicine, vol. 47, no. 6, pp. 1202-1210, 2002.

[6] J. Tsao, P. Boesiger, and K. P. Pruessmann, "k-t BLAST and k-t SENSE: Dynamic MRI with high frame rate exploiting spatiotempora correlations," Magnetic Resonance in Medicine, vol. 50, no. 5, pp. 1031-1042, Nov 2003.

[7] B. Madore, "UNFOLD-SENSE: A parallel MRI method with selfcalibration and artifact suppression," Magnetic Resonance in Medicine, vol. 52, no. 2, pp. 310-320, Aug 2004.

[8] Emmanuel Candes, Justin Romberg, and Terence Tao, "Robust uncertainty principles: Exact signal reconstruction from highly incomplete frequency information," IEEE Transactions on Information Theory, vol. 52, pp. 489-509, 2006.

[9] David Donoho, "Compressed sensing," IEEE Transactions on Information Theory, vol. 52, pp. 1289-1306, 2006.

[10] Joel Tropp and Anna Gilbert, "Signal recovery from random measurements via orthogonal matching pursuit," IEEE Transactions on Information Theory, vol. 53, pp. 4655-4666, 2007.

[11] Michael Lustig, David Donoho, and John M. Pauly, "Sparse MRI: The application of compressed sensing for rapid MR imaging," Magnetic Resonance in Medicine, vol. 58, pp. 1182-1195, 2007

[12] Hong Jung, Kyunghyun Sung, Krishna S. Nayak, Eung Yeop Kim, and Jong Chul Ye, "k-t FOCUSS: A general compressed sensing framework for high resolution dynamic MRI," Magnetic Resonance in Medicine, vol. 61, pp. $103-116,2009$.

[13] Nguyen Linh-Trung, Dinh Van Phong, Z. Hussain, H.T. Huynh, V. Morgan, and J. Gore, "Compressed sensing using chaos filters," in Australian Telecommunication Networks and Applications Conference (ATNAC 2008), 2008, pp. 219-223.

[14] Peter Stavroulakis, Ed., Chaos Applications in Telecommunications, CRC Press.

[15] Emmanuel Candes and Justin Romberg, "Sparsity and incoherence in compressive sampling,” Inverse Problems, vol. 23, pp.969-985, 2007.

[16] Gilles Puy, Yves Wiaux, Rolf Gruetter, Jean-Philippe Thiran, Spread spectrum for accelerated acquisition in magnetic resonance imaging, IEEE Trans. Image Process, 2009.

[17] Tran Duc Tan, Dinh Van Phong, Truong Minh Chinh, and Nguyen LinhTrung, "Accelerated Parallel Magnetic Resonance Imaging with MultiChannel Chaotic Compressed Sensing," 2010 International Conference on Advanced Technologies for Communications ATC 2010, HCM Vietnam, Oct. 2010, pp. 146-151.

[18] D. V. Phong, N. L, Trung, T. D. Tan, H. V. Le, Minh. N. Do, "Fast image acquisition in magnetic resonance imaging by chaotic compressed sensing," 8th IEEE International Symposium on Biomedical Imaging (ISBI'11), March 30 - April 2, 2011.

[19] A. Bilgin, Y. Kim, H. G. Lalgudi, T. P. Trouard, and M. I. Altbach, "Parallel magnetic resonance imaging using compressed sensing," in Proceedings of the SPIE on Applications of Digital Image Processing XXXI, A. G. Tescher, Ed., vol. 7073, 2008.

[20] "SENSE: sensitivity-encoding MRI Matlab tools." [Online]. Available:http://www.nmr.mgh.harvard.edu/ fhlin/tool sense.htm 